\title{
Elevated levels of soluble ST2 but not galectin-3 are associated with increased risk of mortality in hemodialysis patients
}

\author{
Ae Jin Kim ${ }^{1,2}$, Han Ro ${ }^{1,2}$, Hyunsook Kim ${ }^{3}$, Kwang-Pil Ko ${ }^{4}$, Jae Hyun Chang ${ }^{1,2}$, Hyun Hee Lee ${ }^{1,2}$, \\ Wookyung Chung ${ }^{1,2}$, Ji Yong Jung ${ }^{1,2,3}$ \\ ${ }^{1}$ Division of Nephrology, Department of Internal Medicine, Gachon University Gil Medical Center, Incheon, Republic of Korea \\ ${ }^{2}$ Department of Internal Medicine, Gachon University College of Medicine, Incheon, Republic of Korea \\ ${ }^{3}$ Department of Health Sciences and Technology, Gachon University, Incheon, Republic of Korea \\ ${ }^{4}$ Department of Preventive Medicine, Gachon University College of Medicine, Incheon, Republic of Korea
}

Background: The soluble forms of suppression of tumorigenicity-2 (ST2) and galectin-3 have been proposed as novel biomarkers for cardiac fibrosis and heart failure, as well as predictors of cardiovascular events and mortality. However, there are limited data on the association between soluble ST2 and galectin-3 and clinical outcomes in patients with kidney failure on replacement therapy. To determine this, we examined the associations between soluble ST2 and galectin- 3 and all-cause mortality and cardiovascular events in patients on hemodialysis.

Methods: This study included maintenance hemodialysis patients (over 18 years old) who consented to preserve their serum in the Biobank at our institution between March 2014 and March 2015. We used Cox proportional hazards regression analysis to evaluate the associations between soluble ST2, galectin-3 levels, and clinical outcomes. The primary outcome was all-cause mortality, the secondary outcome was cardiovascular disease, and patients were followed for both outcomes until March 2018.

Results: A total of 296 patients were analyzed in this study. The mean age was $57 \pm 13$ years, and $53.0 \%$ were male. Serum concentration of soluble ST2 was significantly associated with higher mortality, after adjustment for confounding factors, but was not associated with cardiovascular disease. Serum galectin-3 level was not independently associated with either outcome after adjustment.

Conclusion: Elevated soluble ST2 is independently associated with an increased risk of mortality, but not with cardiovascular disease, in patients on hemodialysis. Elevated galectin-3 was not associated with mortality or cardiovascular disease.

Keywords: Cardiovascular diseases, Galectin 3, Hemodialysis, Mortality, Soluble ST2

Received: July 23, 2020; Revised: November 4, 2020; Accepted: December 1, 2020

Editor: Chan-Duck Kim, Kyungpook National University, Daegu, Republic of Korea

Correspondence: Ji Yong Jung

Division of Nephrology, Department of Internal Medicine, Gachon University Gil Medical Center, Gachon University College of Medicine, 21 Namdong-daero 774 beon-gil, Namdong-gu, Incheon 21565, Republic of Korea. E-mail: jyjung@gachon.ac.kr

ORCID: https://orcid.org/0000-0003-1271-8012

Copyright ( 2021 by The Korean Society of Nephrology

(a) This is an Open Access article distributed under the terms of the Creative Commons Attribution Non-Commercial and No Derivatives License (http:// creativecommons.org/licenses/by-nc-nd/4.0/) which permits unrestricted non-commercial use, distribution of the material without any modifications, and reproduction in any medium, provided the original works properly cited. 


\section{Introduction}

The incidence and prevalence of kidney failure with replacement therapy (KFRT) are both on the increase, and this constitutes a major public health challenge worldwide [1-3]. Although the mortality rate of patients with KFRT has decreased over the past two decades, both morbidity and mortality remain high; the adjusted survival for incident hemodialysis (HD) patients three years after the onset of KFRT is $57 \%[1,2,4,5]$. Cardiovascular disease (CVD) is common in these patients and contributes significantly to mortality, accounting for nearly $50 \%$ of all deaths [1]. These poor survival trends have led to the need for risk stratification and strategies to improve outcomes in patients with KFRT.

Soluble suppression of tumorigenicity-2 (sST2), a member of the interleukin (IL)-1 receptor family, is thought to be involved in inflammation, myocardial hypertrophy, and fibrosis by neutralizing the effect of IL-33 [6]. Galectin-3 is a $\beta$-galactoside-binding lectin that is thought to be involved in inflammation, the induction of cardiac fibrosis, and ventricular remodeling [7]. Recent studies have suggested sST2 and galectin-3 as novel biomarkers of heart failure (HF) [8] and independent predictors of CVD and mortality in the general population $[6,9]$. Both markers have been approved by the U.S. Food and Drug Administration for clinical use [8]. Some studies have reported that their levels are significantly associated with all-cause mortality in patients with chronic kidney disease (CKD) [10,11].

However, few studies have investigated these biomarkers in patients with KFRT, which has limited their prognostic value. Therefore, in this study, we aimed to investigate whether SST2 and galectin-3 predict adverse outcomes and provide additional prognostic value in maintenance HD patients.

\section{Methods}

\section{Study population}

This study involved participants from a prospective observational cohort of prevalent HD patients at Gachon University Gil Medical Center in Incheon, Republic of Korea. This cohort included patients who were $>18$ years of age and consented to preserve their serum in the Biobank at our institution. We excluded patients with insufficient clinical data, any physical, mental, or medical condition that prohibited the ability to provide informed consent, and those who withdrew consent before follow-up blood analysis, or who declined to store their blood samples. Under the Biobank study protocol, blood samples were collected from each patient at baseline and then yearly for up to 5 years. Predialysis blood samples were collected according to a standardized protocol. Specimens were processed on refrigerated packs on the day of collection and transported to the Biobank, where they were aliquoted and stored at $-80^{\circ} \mathrm{C}$. All patients were treated with a 4-hour HD session (5008S; Fresenius Medical Care, St. Wendel, Germany) twice ( $\mathrm{n}=$ $5)$ or thrice $(n=291)$ per week using high-flux polysulfone membranes (FX CorDiax 60; Fresenius Medical Care). Patients were enrolled for one calendar year between March 2014 and March 2015 and were followed until March 2018. Patients were censored at the time of kidney transplantation ( $\mathrm{n}=20)$, transfer to other dialysis centers $(\mathrm{n}=28)$, loss to follow-up ( $n=15)$, or at the end of follow-up (March 27, 2018). This study adheres to the Declaration of Helsinki and was approved by the Institutional Review Board at the Gachon University Gil Medical Center (GBIRB2018-224). Written informed consent was obtained from all participants. Biospecimens were provided by Gachon University Gil Medical Center Biobank.

\section{Parameters}

All demographics, clinical data, comorbidities, laboratory values, and medications were collected at study enrollment from the participant's medical record by a well-trained study coordinator. Baseline demographic and clinical characteristics were collected as follows: age, sex, body mass index (BMI), and pre- and postdialysis systolic blood pressure and diastolic blood pressure. We identified comorbidities, including hypertension, diabetes mellitus (DM), ischemic heart disease such as angina pectoris and myocardial infarction, HF, transient ischemic attack (TIA), and stroke. Angina pectoris and myocardial infarction were defined as the presence of coronary artery disease as documented by angiography, or an acute coronary syndrome or angina requiring percutaneous coronary intervention or coronary artery bypass grafting surgery. Stroke and TIA were defined as cases where magnetic 
resonance imaging was performed in patients with suspected symptoms and diagnosed by a neurologist. Systolic HF was defined as left ventricular ejection fraction of $<40 \%$ and diastolic HF was defined as E/é $>15$. Laboratory data included hemoglobin ( $\mathrm{Hb})$, white blood cells, platelets, serum creatinine, blood urea nitrogen, albumin, protein, calcium, phosphorus, alkaline phosphatase, uric acid, total cholesterol, $\mathrm{HbAlc}$, and high-sensitivity C-reactive protein (hs-CRP). Anemia was defined as $\mathrm{Hb}$ of $<10 \mathrm{~g} / \mathrm{dL}$. Hypoalbuminemia was defined as serum albumin of $<3.5$ $\mathrm{g} / \mathrm{dL}$. Hypocalcemia was defined as serum total calcium of $<8.2 \mathrm{mg} / \mathrm{dL}$. Hyperphosphatemia was defined as serum phosphorus of $>4.7 \mathrm{mg} / \mathrm{dL}$. Medication data included the use of renin-angiotensin system (RAS) blockers, calcium channel blockers (CCB), $\beta$-blockers, diuretics, and antiplatelet agents.

Serum sST2 and galectin-3 levels $(\mathrm{ng} / \mathrm{mL})$ were measured using commercial quantitative enzyme-linked immunosorbent assay kits DST200 and DGAL30, respectively (Quantikine, R\&D Systems, Minneapolis, MN, USA).

\section{Outcomes}

The primary outcome was all-cause mortality. The secondary outcome was cardiovascular events (CVD), defined as unstable angina pectoris, acute myocardial infarction, TIA, stroke, and HF.

\section{Statistical analyses}

Continuous variables are presented as the mean \pm standard deviation (SD) or median (interquartile range), as appropriate. Categorical data are presented as absolute values and percentage frequency. For continuous variables, between-group comparisons were performed using Student $\mathrm{t}$ tests; for categorical variables, chi-square tests were used. Receiver operating characteristic (ROC) curve analyses were performed to identify the optimal cut-off values for sST2 and galectin-3 with the highest sum of sensitivity and specificity for mortality. Logistic regression analysis was used to demonstrate the association between sST2, galectin-3, and underlying HF. The Cox proportional hazard regression model was used to investigate the prognostic utility of these two biomarkers for predicting mortality and CVD. For Cox analyses, log-transformed sST2 and galectin-3 were used and hazard ratio (HR) refers to a 1 SD rise in log-transformed units. Covariates were adjusted in four different models. The first model was adjusted for age and sex. The second was additionally adjusted for smoking status, HD duration, $\mathrm{DM}$, hypertension, and underlying CVD, including angina pectoris, myocardial infarction, TIA, stroke, and HF. The third was then adjusted for medications (RAS blockers, CCB, $\beta$-blockers, diuretics, and antiplatelet agents), and the fourth model was further adjusted for laboratory variables (anemia, hypoalbuminemia, hypocalcemia, hyperphosphatemia, and hs-CRP). We further examined the interaction between sST2 and several risk factors (age, sex, DM, hypertension, previous CVD, anemia, hypoalbuminemia, and use of RAS blockers and $\beta$-blockers) by including interaction terms in the models. Interaction terms were created by multiplying each factor by sST2. The likelihood ratio test was used to test the significance of the interaction terms. We showed the effect modification of sST2 and galectin-3 for mortality and CVD in prespecified subgroups: age ( $<70$ years vs. $\geq 70$ years), sex (female vs. male), DM, hypertension, previous CVD, anemia (Hb of $<10 \mathrm{~g} / \mathrm{dL}$ vs. $\geq 10 \mathrm{~g} / \mathrm{dL}$ ), hypoalbuminemia (albumin of $<3.5 \mathrm{~g} / \mathrm{dL}$ vs. $\geq 3.5 \mathrm{~g} / \mathrm{dL}$ ), and use of RAS blockers and $\beta$-blockers. Statistical analyses were performed using IBM SPSS version 22.0 (IBM Corp., Armonk, NY, USA). All analyses were two-sided and p-values of $<0.05$ were considered statistically significant.

\section{Results}

\section{Patient characteristics (baseline characteristics)}

The baseline characteristics of the study population are presented in Table 1. A total of 296 patients enrolled in this study. The mean age was $57 \pm 13$ years and $53.0 \%$ were male. In this cohort, $45.6 \%$ of the patients were diabetic, $86.5 \%$ were hypertensive, $23.6 \%$ had ischemic heart disease, $10.8 \%$ had TIA or stroke, and $35.8 \%$ had HF. Survivors were younger, less likely to have DM or HF, more likely to take statins, and had higher creatinine, phosphorus, and albumin and lower SST2 and galectin-3 levels than non-survivors. The median serum sST2 and galectin-3 level were higher in non-survivors than in survivors: sST2, $21.190 \mathrm{ng} / \mathrm{mL}$ (interquartile range [IQR], 16.208-29.176) vs. $28.200 \mathrm{ng} / \mathrm{mL}$ (IQR, 20.851-37.763), $\mathrm{p}=0.003$ (Table 1); galectin-3, 34.957 ng/mL (IQR, 28.413-41.908) vs. 38.619 ng/mL (IQR, 31.013- 
Table 1. Baseline characteristics

\begin{tabular}{|c|c|c|c|c|}
\hline Characteristic & Total $(n=296)$ & Survivor $(n=260)$ & Non-survivor $(n=36)$ & p-value \\
\hline Age (yr) & $57 \pm 13$ & $56 \pm 13$ & $64 \pm 13$ & $0.001 *$ \\
\hline Male sex & $157(53.0)$ & $138(53.1)$ & $19(52.8)$ & 0.973 \\
\hline BMI $\left(\mathrm{kg} / \mathrm{m}^{2}\right)$ & $22.5 \pm 3.8$ & $22.5 \pm 3.9$ & $21.9 \pm 3.2$ & 0.383 \\
\hline Pre-HD SBP (mmHg) & $146 \pm 26$ & $147 \pm 27$ & $142 \pm 22$ & 0.249 \\
\hline Pre-HD DBP (mmHg) & $70 \pm 14$ & $71 \pm 14$ & $62 \pm 11$ & $<0.001 *$ \\
\hline Post-HD SBP (mmHg) & $131 \pm 24$ & $131 \pm 24$ & $127 \pm 23$ & 0.353 \\
\hline Post-HD DBP (mmHg) & $69 \pm 41$ & $68 \pm 13$ & $79 \pm 113$ & 0.584 \\
\hline HD duration (mo) & $48.5(18.0-75.8)$ & $47.5(17.0-74.8)$ & 55.0 (33.5-102.0) & 0.318 \\
\hline \multicolumn{5}{|l|}{ Comorbidity } \\
\hline Hypertension & $256(86.5)$ & $224(86.2)$ & $32(88.9)$ & 0.799 \\
\hline $\mathrm{DM}$ & $135(45.6)$ & $113(43.5)$ & $22(61.1)$ & $0.046 *$ \\
\hline IHD & $70(23.6)$ & 57 (21.9) & $13(36.1)$ & 0.060 \\
\hline Stroke or TIA & $32(10.8)$ & $25(9.6)$ & 7 (19.4) & 0.086 \\
\hline Heart failure & $106(35.8)$ & $87(33.5)$ & $19(52.8)$ & $0.023 *$ \\
\hline \multicolumn{5}{|l|}{ Laboratory } \\
\hline Hemoglobin (g/dL) & $11.0 \pm 5.6$ & $11.1 \pm 6.0$ & $10.0 \pm 1.4$ & 0.295 \\
\hline $\operatorname{WBC}\left(\times 10^{3} / \mathrm{mm}\right)$ & $5.82 \pm 2.18$ & $5.79 \pm 2.13$ & $6.03 \pm 2.47$ & 0.540 \\
\hline $\operatorname{PLT}\left(\times 10^{3} / \mathrm{mm}\right)$ & $191(150-232)$ & $191(154-234)$ & $187(112-228)$ & 0.177 \\
\hline BUN (mg/dL) & $55.1 \pm 16.1$ & $55.0 \pm 15.7$ & $55.5 \pm 18.4$ & 0.856 \\
\hline Creatinine (mg/dL) & $8.3 \pm 2.3$ & $8.5 \pm 2.4$ & $7.4 \pm 1.9$ & $0.012 *$ \\
\hline Calcium (mg/dL) & $8.7 \pm 0.6$ & $8.7 \pm 0.6$ & $8.7 \pm 0.8$ & 0.926 \\
\hline Phosphorus (mg/dL) & $4.6 \pm 1.6$ & $4.7 \pm 1.6$ & $4.0 \pm 1.5$ & $0.024 *$ \\
\hline $\operatorname{ALP}(I U / m L)$ & $83.0(66.0-110.8)$ & $82.0(65.0-107.0)$ & $93.5(76.5-128.5)$ & 0.323 \\
\hline Albumin (g/dL) & $3.9 \pm 0.4$ & $3.9 \pm 0.4$ & $3.7 \pm 0.4$ & $0.003 *$ \\
\hline Protein $(\mathrm{g} / \mathrm{dL})$ & $6.9 \pm 0.5$ & $6.9 \pm 0.5$ & $6.8 \pm 0.6$ & 0.127 \\
\hline Uric acid (mg/dL) & $7.0 \pm 1.4$ & $7.1 \pm 1.4$ & $6.6 \pm 1.4$ & 0.054 \\
\hline $\mathrm{TC}(\mathrm{mg} / \mathrm{dL})$ & $137 \pm 29$ & $137 \pm 29$ & $139 \pm 29$ & 0.635 \\
\hline HbA1c (\%) & $6.2 \pm 4.2$ & $6.2 \pm 4.5$ & $6.0 \pm 1.7$ & 0.797 \\
\hline hs-CRP (mg/dL) & $0.13(0.05-0.45)$ & $0.12(0.04-0.39)$ & $0.24(0.10-1.04)$ & 0.628 \\
\hline $\mathrm{sST} 2(\mathrm{ng} / \mathrm{mL})$ & $22.074(16.515-30.768)$ & $21.190(16.208-29.176)$ & $28.200(20.851-37.763)$ & $0.003 *$ \\
\hline Galectin-3 (ng/mL) & 35.609 (28.534-41.981) & 34.957 (28.413-41.908) & 38.619 (31.013-45.576) & $0.044 *$ \\
\hline \multicolumn{5}{|l|}{ Medication } \\
\hline RAS blockers & $143(48.3)$ & $124(47.7)$ & $19(52.8)$ & 0.567 \\
\hline $\mathrm{CCB}$ & $148(50.0)$ & $128(49.2)$ & $20(55.6)$ & 0.477 \\
\hline$\beta$-blockers & $120(40.5)$ & $104(40.0)$ & $16(44.4)$ & 0.611 \\
\hline Diuretics & $159(53.7)$ & $144(55.4)$ & $15(41.7)$ & 0.122 \\
\hline Anti-platelets & $250(84.5)$ & $221(85.0)$ & $29(80.6)$ & 0.490 \\
\hline
\end{tabular}

Data are expressed as the mean \pm standard deviation, median (interquartile range), or number of patients (\%).

ALP, alkaline phosphatase; BMI, body mass index; BUN, blood urea nitrogen; CCB, calcium channel blocker; DBP, diastolic blood pressure; DM, diabetes mellitus; HbA1c, hemoglobin A1c; HD, hemodialysis; hs-CRP, high-sensitivity C-reactive protein; IHD, ischemic heart disease; PLT, platelet; RAS, reninangiotensin system; SBP, systolic blood pressure; sST2, soluble suppression of tumorigenicity-2; TC, total cholesterol; TIA, transient ischemic attack; WBC, white blood cell.

$* p<0.05$.

45.576), $\mathrm{p}=0.044$ (Table 1). There were no differences in the HD duration or use of RAS blockers, CCB, $\beta$-blockers, diuretics, and antiplatelet agents between survivors and non-survivors.

\section{Serum soluble suppression of tumorigenicity-2 and galectin-3 levels as predictors of mortality}

During a mean follow-up period of $37.8 \pm 13.9$ months, 36 
patients (12.5\%) died. The main cause of death was infection (9 patients). Other causes included cardiovascular (6), malignancy (4), bleeding (6), aspiration (1), and unknown (10). The predictive value of sST2 and galectin-3 for mortality was evaluated using ROC analysis (Fig. 1). The area under curve of sST2 and galectin-3 for mortality were 0.645 (p = $0.005)$ and $0.578(\mathrm{p}=0.129)$, respectively. The calculated cutoff values of sST2 and galectin-3 for mortality were $22.07 \mathrm{ng} /$ $\mathrm{mL}$ (sensitivity, $75.0 \%$; specificity $53.5 \%$ ) and $35.27 \mathrm{ng} / \mathrm{mL}$ (sensitivity, 66.7\%; specificity, 51.2\%), respectively.

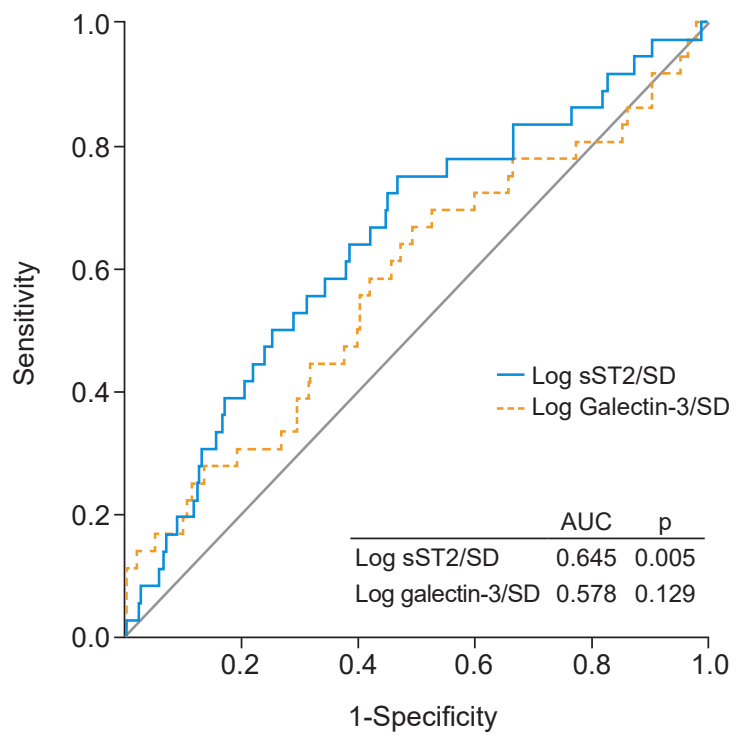

Figure 1. Receiver operating characteristic curve for sST2 and galectin-3 to predict mortality.

AUC, area under curve; SD, standard deviation; sST2, soluble suppression of tumorigenicity- 2 .
In the Cox proportional hazard analysis, serum sST2 was significantly associated with an increased risk of allcause mortality (HR per 1 SD increase of log-transformed sST2, 1.811; 95\% confidence interval [CI], 1.240-2.645; p $=0.002$ ) (Crude in Table 2). After adjusting for covariates, serum sST2 level remained an independent predictor of allcause mortality (HR, 1.589; 95\% CI, 1.016-2.484; $\mathrm{p}=0.042$ ) (Model 4 in Table 2). Because of the significant interaction between sST2 and hypoalbuminemia for all-cause mortality in the multivariable Cox proportional hazard model, the interaction term (sST2*hypoalbuminemia) was included in the final multivariable analysis. Serum sST2 was still associated with all-cause mortality (HR, 2.100; 95\% CI, 1.231-3.580; $\mathrm{p}=0.006$ ) (Model 5 in Table 2).

In contrast to sST2, serum galectin-3 level was not associated with all-cause mortality (HR per 1 SD increase of log-transformed galectin-3, 1.354; 95\% CI, 0.931-1.971; p $=0.113$ ) (Crude in Table 2). After adjustment for covariates, galectin-3 level was still not associated with all-cause mortality (HR per 1 SD increase, 1.450; 95\% CI, 0.926-2.271; $\mathrm{p}=0.105)$ (Model 4 in Table 2).

\section{Serum SST2 and galectin-3 levels as predictors of CVDs}

During the follow-up period, a total of 69 CVDs occurred; of these, 33 events were unstable angina pectoris/myocardial infarction, 35 were HF, and one was TIA/stroke. Using Cox proportional hazard analysis, serum sST2 level was not found to be associated with CVD (HR per 1 SD increase, 1.100; 95\% CI, 0.855-1.414; $\mathrm{p}=0.460$ ) (Crude in Table 3),

Table 2. Adjusted HR for death from any causes based on levels of sST2 and log galectin-3

\begin{tabular}{lclcc}
\hline Adjust model & Log sST2 & $\mathrm{p}$-value & Log galectin-3 & $\mathrm{p}$-value \\
\hline Crude & $1.811(1.240-2.645)$ & $0.002 *$ & $1.354(0.931-1.971)$ & 0.113 \\
Model 1 & $1.804(1.203-2.707)$ & $0.004 *$ & $1.279(0.882-1.854)$ & 0.194 \\
Model 2 & $1.917(1.247-2.947)$ & $0.003 *$ & $1.244(0.861-1.798)$ & 0.246 \\
Model 3 & $1.833(1.192-2.819)$ & $0.006 *$ & $1.337(0.899-1.987)$ & 0.152 \\
Model 4 & $1.589(1.016-2.484)$ & $0.042 *$ & $1.450(0.926-2.271)$ & 0.105 \\
Model 5 & $2.100(1.231-3.580)$ & $0.006 *$ & $\mathrm{NA}$ & $\mathrm{NA}$ \\
\hline
\end{tabular}

Data are expressed as HR $(95 \% \mathrm{Cl})$; HRs are calculated based on per standard deviation increase in log-transformed biomarker level.

Model 1, adjusted for demographics (age and sex); model 2, adjusted for demographics, and comorbidities (model $1+$ smoking status, hemodialysis duration, diabetes mellitus, hypertension, and cardiovascular disease); model 3, adjusted for demographics, comorbidities, and medications (model 2 + renin-angiotensin system blockades, calcium channel blockers, $\beta$-blockers, diuretics, and antiplatelet agents); model 4, adjusted for demographics, comorbidities, medications, and laboratory findings (model $3+$ anemia, hypoalbuminemia, hypocalcemia, hyperphosphatemia, and high-sensitivity C-reactive protein); model 5 , model $4+$ interaction term SST2 $\times$ hypoalbuminemia.

$\mathrm{Cl}$, confidence interval; HR, hazard ratio; NA, not applicable; sST2, soluble suppression of tumorigenicity-2.

${ }^{*} \mathrm{p}<0.05$. 
Table 3. Adjusted HR for incidence of cardiovascular disease based on levels of log sST2 and log galectin-3

\begin{tabular}{lcccc}
\hline Adjust model & Log sST2 & p-value & Log galectin-3 & $p$-value \\
\hline Crude & $1.100(0.855-1.414)$ & 0.460 & $1.042(0.820-1.325)$ & 0.734 \\
Model 1 & $1.055(0.802-1.387)$ & 0.703 & $0.999(0.787-1.267)$ & 0.991 \\
Model 2 & $1.035(0.784-1.367)$ & 0.806 & $0.979(0.768-1.248)$ & 0.863 \\
Model 3 & $1.008(0.765-1.329)$ & 0.954 & $0.984(0.768-1.262)$ & 0.901 \\
Model 4 & $0.993(0.748-1.318)$ & 0.961 & $0.969(0.753-1.247)$ & 0.808 \\
Model 5 & $0.427(0.110-1.655)$ & 0.218 & NA & NA \\
\hline
\end{tabular}

Data are expressed as HR $(95 \% \mathrm{Cl})$; HRs are calculated based on per standard deviation increase in log-transformed biomarker level.

Model 1, adjusted for demographics (age and sex); model 2, adjusted for demographics, and comorbidities (model $1+$ smoking status, hemodialysis duration, diabetes mellitus, hypertension, and cardiovascular disease); model 3, adjusted for demographics, comorbidities, and medications (model 2 + renin-angiotensin system blockades, calcium channel blockers, $\beta$-blockers, diuretics, and antiplatelet agents); model 4, adjusted for demographics, comorbidities, medications, and laboratory findings (model $3+$ anemia, hypoalbuminemia, hypocalcemia, hyperphosphatemia, and high-sensitivity C-reactive protein); model 5, model $4+$ interaction term sST2 $\times$ age and sST2 $\times \beta$-blockers.

$\mathrm{Cl}$, confidence interval; HR, hazard ratio; NA, not applicable; sST2, soluble suppression of tumorigenicity-2.

even after adjustment for covariates (HR per 1 SD increase, 0.427; 95\% CI, 0.110-1.655; $\mathrm{p}=0.218$ ) (Model 5 in Table 3).

Serum galectin-3 level was also not associated with CVD (HR per 1 SD increase, 1.042; 95\% CI, 0.820-1.325; $\mathrm{p}=0.734$ ) (Crude in Table 3), even after adjustment for covariates (HR per 1 SD increase, 0.969; 95\% CI, 0.753-1.247; $\mathrm{p}=0.808$ ) (Model 4 in Table 3).

\section{Subgroup analyses}

In subgroup analysis, we found that hypoalbuminemia modified the association between sST2 and mortality in patients undergoing HD (Fig. 2). When we analyzed the risk of all-cause mortality by Cox proportional hazard analyses of log SST2 per 1 SD increase, in those with hypoalbuminemia, the HR of sST2 was 0.887 (95\% CI, $0.427-1.842 ; \mathrm{p}=0.748$ ) while it was 2.011 (95\% CI, 1.287-3.141; $\mathrm{p}=0.002)$ in those without hypoalbuminemia (Fig. 2). The p-value for this interaction was 0.047 .

The HRs of sST2 for CVD among those with age of $<70$ years and $\geq 70$ years were 0.944 (95\% CI, 0.717-1.243; $\mathrm{p}=0.682)$ and $2.399(95 \% \mathrm{CI}, 1.205-4.773 ; \mathrm{p}=0.013)$ (Supplementary Fig. 1, available online). The p-value for this interaction was 0.010 . The HRs of sST2 for CVD among those without $\beta$-blocker and with $\beta$-blocker were 1.429 (95\% CI, 0.968-2.111; $\mathrm{p}=0.073$ ) and 0.827 (95\% CI, 0.582-1.175; $\mathrm{p}$ $=0.289$ ) (Supplementary Fig. 1). The p-value for interaction was 0.041 . There were no significant interactions between galectin-3 and subgroups for all-cause mortality and CVD (Supplementary Fig. 2, 3; available online).

\section{Association of serum SST2 and galectin-3 with underlying $\mathrm{HF}$}

In a logistic regression analysis, underlying HF (composite of systolic HF and diastolic HF) was not significantly associated with serum sST2 (odds ratio [OR] per 1 SD increase of logtransformed sST2, 1.008; 95\% CI, 0.794-1.278; $\mathrm{p}=0.950)$ (Crude in Supplementary Table 1, available online), or galectin-3 levels (OR per 1 SD increase of log-transformed galectin-3, 0.843; 95\% CI, 0.665-1.067; $\mathrm{p}=0.156$ ) (Crude in Supplementary Table 1). Serum sST2 and galectin-3 levels were still not associated with underlying HF after adjusting for covariates (sST2: OR, 0.987; 95\% CI, 0.737-1.322; $\mathrm{p}=$ 0.929; galectin-3: OR, 0.806; 95\% CI, 0.614-1.058; $\mathrm{p}=0.120$ ) (Model 4 in Supplementary Table 1).

\section{Discussion}

In this study, we investigated the association between two novel biomarkers and clinical outcomes in patients undergoing HD. We showed that the sST2 level was an independent predictor for survival in HD patients, unlike galectin-3. We also demonstrated that elevated sST2 and galectin-3 were not independently predictive of CVD in HD patients.

ST2, a member of the IL-1 receptor family, exists in two main isoforms: SST2 (soluble form) and ST2L (transmembrane form) [12]. The IL-33/ST2 pathway is associated with inflammation and the pathologic process of CVD. In cardiomyocytes and fibroblasts, it is upregulated in response to myocardial stress [13], and has a cardioprotective function, conferring anti- 
fibrotic, anti-hypertrophic, and anti-apoptotic effects to volume-overloaded or injured myocardium [13]. However, sST2, a soluble, truncated form of ST2L, is secreted into the circulation and is believed to function as a decoy receptor for IL-33, inhibiting the effects of IL-33/ST2 signaling, and reducing its various activities, including cardioprotective effects [13]. Serum levels of sST2 are significantly increased in inflammatory diseases, cancer, liver, and cardiac diseases [6,14-16].

To date, the prognostic value of sST2 has been mainly studied in the general population and has been significantly associated with mortality and adverse outcomes in patients with HF [17] and coronary artery disease, including myocardial infarction [18]. Hitherto, studies that evaluated the association of sST2 with kidney function have shown conflicting results. An analysis of the Cardiovascular Health Study cohorts (a community-based multicenter cohort of older adults free of clinical HF) found that SST2 was not associated with kidney function decline or the development of incident estimated glomerular filtration rate (eGFR) $<60 \mathrm{~mL} / \mathrm{min} / 1.73 \mathrm{~m}^{2}$ [19]. Similarly, a study of participants of the Framingham Offspring Study cohort found no significant association between sST2 and risk for incident CKD [20]. However, sST2 was associated with incident albuminuria in a previous study [20], and with the risk of acute kidney injury in patients with ST-segment elevation myocardial infarction [21].

sST2 is a promising prognostic marker for patients with CKD. The recent Seattle Kidney Study (SKS) and Clinical Phenotyping and Resource Biobank Study (C-PROBE) cohort suggested that sST2 was also associated with allcause mortality and a composite of hospitalization and atherosclerotic CVD events [10].

There are limited data on the prognostic value of sST2 in dialysis patients. In our study, elevated sST2 concentration was significantly associated with increased mortality in maintenance HD patients. In a previous study of $414 \mathrm{HD}$ patients, elevated sST2 concentration was an independent predictor of all-cause and cardiovascular mortality [22]. Another recent observational study of 423 HD patients investigated the association of SST2 with allcause mortality and the composite outcome of death and cerebrocardiovascular events [23]. It also suggested that sST2 had an independent and incremental prognostic value for both outcomes [23]. Our data support previous studies that demonstrated an association of sST2 and all-cause mortality. In this regard, sST2 may be used as a prognostic marker for survival in HD patients. In subgroup analysis, the association of sST2 with mortality disappeared in patients with hypoalbuminemia. Because hypoalbuminemia is a significant predictor of mortality in this population, it might attenuate the association between sST2 and mortality.

However, the risk of CVD was not associated with sST2 levels in our study. Recent studies on the general population showed an association of sST2 with CVD [24]. However, in a study of 883 CKD patients, sST2 concentration was not associated with atherosclerotic CVD [10]. There is no previous study showing an association of SST2 with solely CVD in HD patients. Patients with CKD have unique CVD risk factors, such as metabolic abnormalities, disordered mineral metabolism, and accumulation of uremic toxins, which may impact the association between sST2 and CVD. In addition, previous studies suggest that sST2 may reflect hemodynamic alterations and inflammatory status in the general population $[25,26]$; therefore, more complex underlying pathophysiology could also impact the relationship between sST2 and CVD in this population, including the larger hemodynamic burden and the increased inflammatory environment of HD patients. In subgroup analysis, sST2 was associated with the risk of CVD in older patients (aged $\geq 70$ years). There were fewer patients in the older group, but the CVD event rate was high. This result could be attributable to the small size of this high-risk group. Further studies are needed to investigate associations with SST2 and CVD in this population.

Galectin-3 is a 29 - to $35-\mathrm{kDa}$ protein and belongs to the family of $\beta$-galactoside-binding lectins [27]. It plays a role in embryonic development and promotes fibrosis and inflammation [28]. Previous studies have shown that galectin-3 is associated with cardiac fibrosis, ventricular remodeling, and dysfunction $[7,29]$. In support of these mechanisms, many clinical studies have demonstrated that galectin-3 has a predictive value for the diagnosis and prognosis of acute and chronic HF [30]. Galectin-3 is also associated with CVD and all-cause mortality in the general population [31,32].

In the kidney, galectin-3 is also a profibrotic agent and could be considered as a marker of fibrosis [29]. Serum galectin-3 levels are inversely correlated with kidney function in the general population [33] and in patients with HF [34] or CKD [10]. Clinical studies have shown that 


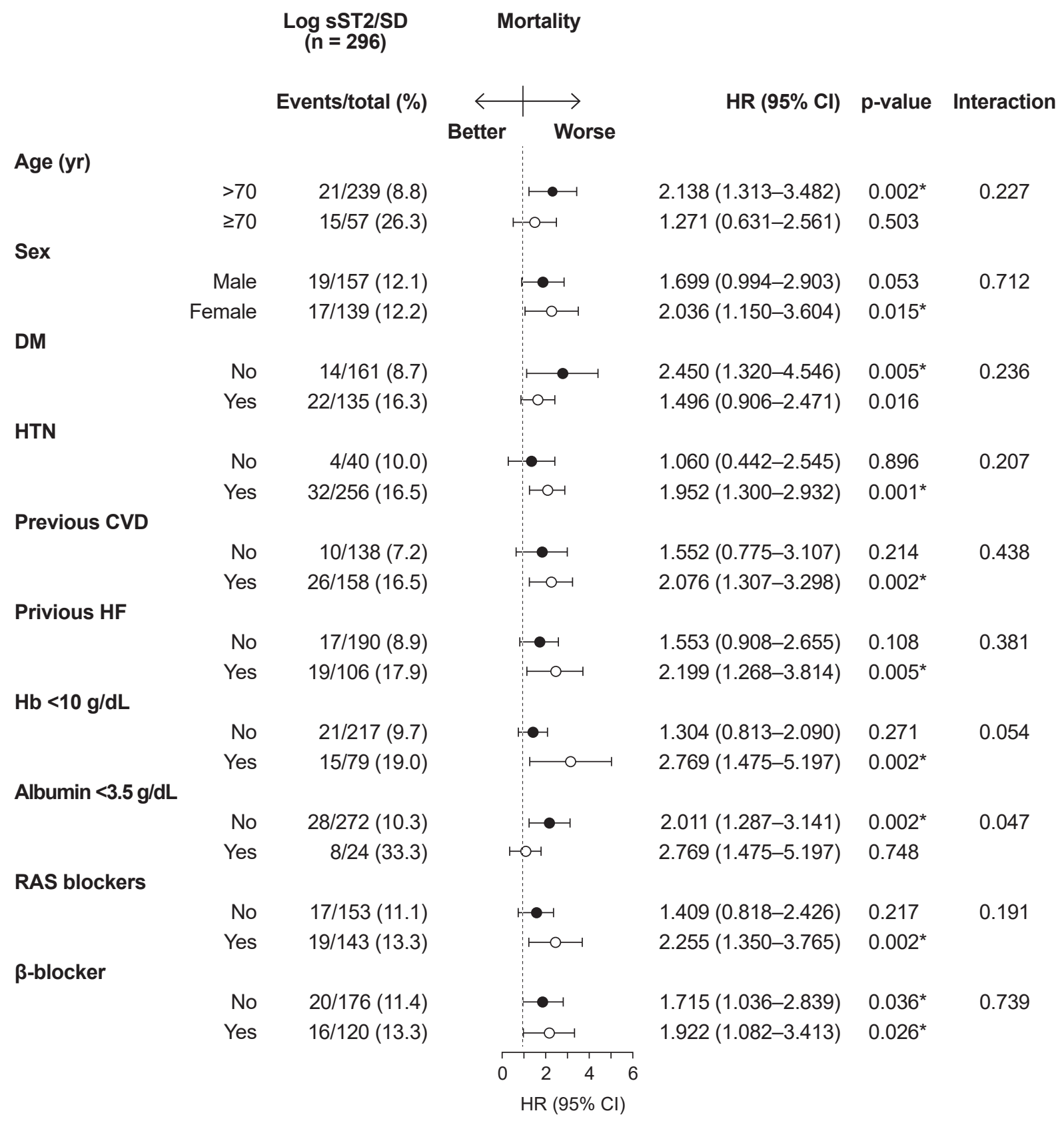

Figure 2. Association of log sST2 and mortality in subgroups of patients (per SD increase).

$\mathrm{Cl}$, confidence interval; CVD, cardiovascular disease; DM, diabetes mellitus; Hb, hemoglobin; HF, heart failure; HR, hazard ratio; HTN, hypertension; RAS, renin-angiotensin system; SD, standard deviation; sST2, soluble suppression of tumorigenicity-2.

$* p<0.05$.

elevated galectin-3 levels are associated with increased risk of rapid decline in eGFR [35] and incident CKD in the general population $[35,36]$. In patients with CKD, higher circulating galectin-3 concentration is significantly associated with greater mortality $[10,11]$.

In our study, however, higher galectin-3 level was not significantly associated with all-cause mortality in HD patients. There are few studies to demonstrate the association of galectin-3 with risk for mortality in patients undergoing HD, and any such results are controversial $[11,23,37,38]$. In the analysis of the German Diabetes Dialysis Study (4D study), galectin-3 concentration was 
not associated with all-cause mortality after adjusting for confounders [11]. Even in the meta-analysis from four studies, there was no statistical difference between galectin-3 and the risk of all-cause mortality in HD patients [37]. However, Obokata et al. [23] reported galectin-3 as an independent predictor of all-cause death in patients receiving HD, and Hogas et al. [38] also found it to be an independent predictor of mortality.

Previous studies demonstrated that the concentration of galectin-3 was inversely correlated with eGFR and was further elevated in patients on dialysis [10,11]. In a $4 \mathrm{D}$ cohort of HD patients, the mean galectin-3 concentration was $54 \mathrm{ng} / \mathrm{mL}$, compared to $11-14 \mathrm{ng} / \mathrm{mL}$ for the general population [11]. In our study of HD patients, the mean galectin-3 concentration was $35.5 \mathrm{ng} / \mathrm{mL}$. Furthermore, in previous studies of congestive HF, adding kidney function to regression analysis substantially attenuated the prognostic power of galectin-3, suggesting that elevated levels of galectin-3 could compromise the prognostic value in this population.

In the present study, galectin-3 concentrations are not associated with CVD in HD patients. Previous studies associating galectin-3 with CVD are limited. In the German $4 \mathrm{D}$ study, galectin-3 concentration was a predictor of CVD in HD patients [11], and Obokata et al. [23] reported that galectin-3 was significantly associated with a composite of deaths and cerebrocardiovascular events. Further studies are needed to elucidate the relationship between galectin-3 and cardiovascular outcomes.

Our study has several limitations. First, we did not exclude patients with underlying HF, although our multivariable logistic regression analysis shows that there is no association between sST2 and galectin-3 concentration with underlying HF in patients undergoing HD. Second, we measured the sST2 and galectin-3 levels from frozen samples rather than fresh ones; bias from using frozen samples cannot be excluded. Third, it is possible that elevated galectin-3 and sST2 concentrations reflect decreased kidney function in this population, and we cannot determine mechanisms or causality of the association from this observational study. Fourth, there was no residual kidney function data and this could not be adjusted. Fifth, this study is a single-center study with a single ethnic group. Thus, further studies with other centers and ethnic groups are needed, to generalize our findings. Finally, despite considering multiple risk factors, it is possible that we may have omitted residual confounding from the analysis.

In conclusion, sST2 is a significant predictor of all-cause mortality in HD patients, but galectin-3 is not, and neither is associated with CVD. Our study highlights the need for more research to elucidate the mechanism causing the observed elevations in sST2, specifically in the vulnerable HD population. Further studies are also needed to validate the association of sST2 and galectin-3 with clinical outcomes.

\section{Conflicts of interest}

All authors have no conflicts of interest to declare.

\section{Funding}

This study was supported by the Young Investigator Research Grant from the Korean Society of Nephrology in 2018.

\section{Acknowledgments}

We appreciate the cooperation of Gachon University Gil Medical Center Biobank for giving us the biospecimens and the data of enrolled patients (No: GBB2014-04).

\section{Authors' contributions}

Conceptualization: JYJ

Data curation: AJK, JYJ, HK

Formal analysis: AJK, JYJ, KPK

Funding acquisition: $\mathrm{AJK}$

Investigation: AJK, HK

Methodology: JYJ

Writing-original draft: AJK, JYJ

Writing-review \& editing: AJK, JYJ, HR, JHC, HHL, WC

All authors read and approved the final manuscript.

\section{ORCID}

Ae Jin Kim, https://orcid.org/0000-0001-5017-8649

Han Ro, https://orcid.org/0000-0001-7170-0571

Hyunsook Kim, https://orcid.org/0000-0002-0674-2713

Kwang-Pil Ko, https://orcid.org/0000-0002-7788-2887

Jae Hyun Chang, https://orcid.org/0000-0003-3947-0715

Hyun Hee Lee, https://orcid.org/0000-0003-2200-5948 


\section{Wookyung Chung, https://orcid.org/0000-0001-7657-130X Ji Yong Jung, https://orcid.org/0000-0003-1271-8012}

\section{References}

1. Saran R, Robinson B, Abbott KC, et al. US renal data system 2018 annual data report: epidemiology of kidney disease in the United States. Am J Kidney Dis 2019;73(3 Suppl 1):A7-A8.

2. Pippias M, Stel VS, Abad Diez JM, et al. Renal replacement therapy in Europe: a summary of the 2012 ERA-EDTA Registry Annual Report. Clin Kidney J2015;8:248-261.

3. Jin DC, Yun SR, Lee SW, et al. Lessons from 30 years' data of Korean end-stage renal disease registry, 1985-2015. Kidney Res Clin Pract 2015;34:132-139.

4. Wang V, Vilme H, Maciejewski ML, Boulware LE. The economic burden of chronic kidney disease and end-stage renal disease. Semin Nephrol 2016;36:319-330.

5. Choi H, Kim M, Kim H, et al. Excess mortality among patients on dialysis: comparison with the general population in Korea. Kidney Res Clin Pract 2014;33:89-94.

6. Weinberg EO, Shimpo M, Hurwitz S, Tominaga S, Rouleau JL, Lee RT. Identification of serum soluble ST2 receptor as a novel heart failure biomarker. Circulation 2003;107:721-726.

7. Sharma UC, Pokharel S, van Brakel TJ, et al. Galectin-3 marks activated macrophages in failure-prone hypertrophied hearts and contributes to cardiac dysfunction. Circulation 2004;110:3121-3128.

8. de Boer RA, Daniels LB, Maisel AS, Januzzi JL Jr. State of the art: newer biomarkers in heart failure. Eur J Heart Fail 2015;17:559569.

9. Jansen H, Koenig W, Jaensch A, et al. Prognostic utility of galectin-3 for recurrent cardiovascular events during long-term follow-up in patients with stable coronary heart disease: results of the KAROLA study. Clin Chem 2016;62:1372-1379.

10. Tuegel C, Katz R, Alam M, et al. GDF-15, galectin 3, soluble ST2, and risk of mortality and cardiovascular events in CKD. Am J Kidney Dis 2018;72:519-528.

11. Drechsler C, Delgado G, Wanner C, et al. Galectin-3, renal function, and clinical outcomes: results from the LURIC and 4D studies. J Am Soc Nephrol 2015;26:2213-2221.

12. Kakkar R, Lee RT. The IL-33/ST2 pathway: therapeutic target and novel biomarker. Nat Rev Drug Discov 2008;7:827-840.

13. Pascual-Figal DA, Januzzi JL. The biology of ST2: the International ST2 Consensus Panel. Am J Cardiol 2015;115(7 Suppl):3B-7B.

14. Bergis D, Kassis V, Radeke HH. High plasma sST2 levels in gastric cancer and their association with metastatic disease. Cancer Biomark 2016;16:117-125.

15. Yang ZP, Ling DY, Xie YH, et al. The association of serum IL-33 and sST2 with breast cancer. Dis Markers 2015;2015:516895.

16. Gao S, Huan SL, Han LY, et al. Overexpression of serum sST2 is associated with poor prognosis in acute-on-chronic hepatitis B liver failure. Clin Res Hepatol Gastroenterol 2015;39:315-323.

17. Januzzi JL, Mebazaa A, Di Somma S. ST2 and prognosis in acutely decompensated heart failure: the International ST2 Consensus Panel. Am J Cardiol 2015;115(7 Suppl):26B-31B.

18. Sabatine MS, Morrow DA, Higgins LJ, et al. Complementary roles for biomarkers of biomechanical strain ST2 and N-terminal prohormone B-type natriuretic peptide in patients with STelevation myocardial infarction. Circulation 2008;117:19361944.

19. Bansal N, Katz R, Seliger S, et al. Galectin-3 and soluble ST2 and kidney function decline in older adults: the Cardiovascular Health Study (CHS). Am J Kidney Dis 2016;67:994-996.

20. Ho JE, Hwang SJ, Wollert KC, et al. Biomarkers of cardiovascular stress and incident chronic kidney disease. Clin Chem 2013; 59:1613-1620.

21. Tung YC, Chang CH, Chen YC, Chu PH. Combined biomarker analysis for risk of acute kidney injury in patients with ST-segment elevation myocardial infarction. PLoS One 2015;10:e125282.

22. Zhang Z, Shen B, Cao X, et al. Increased soluble suppression of tumorigenicity 2 level predicts all-cause and cardiovascular mortality in maintenance hemodialysis patients: a prospective cohort study. Blood Purif 2017;43:37-45.

23. Obokata M, Sunaga H, Ishida $H$, et al. Independent and incremental prognostic value of novel cardiac biomarkers in chronic hemodialysis patients. Am Heart J2016;179:29-41.

24. Jha D, Goenka L, Ramamoorthy T, Sharma M, Dhandapani VE, George M. Prognostic role of soluble ST2 in acute coronary syndrome with diabetes. Eur J Clin Invest 2018;48:e12994.

25. Broch K, Andreassen AK, Ueland T, et al. Soluble ST2 reflects hemodynamic stress in non-ischemic heart failure. Int J Cardiol 2015;179:378-384.

26. Mueller T, Jaffe AS. Soluble ST2: analytical considerations. Am J Cardiol 2015;115(7 Suppl):8B-21B.

27. Yang RY, Rabinovich GA, Liu FT. Galectins: structure, function and therapeutic potential. Expert Rev Mol Med 2008;10:e17.

28. Dumic J, Dabelic S, Flögel M. Galectin-3: an open-ended story. Biochim Biophys Acta 2006;1760:616-635.

29. Calvier L, Miana M, Reboul P, et al. Galectin-3 mediates aldosterone-induced vascular fibrosis. Arterioscler Thromb Vasc 


\section{Biol 2013;33:67-75.}

30. de Boer RA, Voors AA, Muntendam P, van Gilst WH, van Veldhuisen DJ. Galectin-3: a novel mediator of heart failure development and progression. Eur J Heart Fail 2009;11:811-817.

31. Imran TF, Shin HJ, Mathenge N, et al. Meta-analysis of the usefulness of plasma galectin-3 to predict the risk of mortality in patients with heart failure and in the general population. Am J Cardiol 2017;119:57-64.

32. Daniels LB, Clopton P, Laughlin GA, Maisel AS, Barrett-Connor E. Galectin-3 is independently associated with cardiovascular mortality in community-dwelling older adults without known cardiovascular disease: The Rancho Bernardo Study. Am Heart J 2014;167:674-682.

33. de Boer RA, van Veldhuisen DJ, Gansevoort RT, et al. The fibrosis marker galectin-3 and outcome in the general population. $J$ Intern Med 2012;272:55-64.
34. de Boer RA, Lok DJ, Jaarsma T, et al. Predictive value of plasma galectin-3 levels in heart failure with reduced and preserved ejection fraction. Ann Med 2011;43:60-68.

35. O'Seaghdha CM, Hwang SJ, Ho JE, Vasan RS, Levy D, Fox CS Elevated galectin-3 precedes the development of CKD. J Am Soc Nephrol 2013;24:1470-1477.

36. Rebholz CM, Selvin E, Liang M, et al. Plasma galectin-3 levels are associated with the risk of incident chronic kidney disease. Kidney Int 2018;93:252-259.

37. Zhang T, Cao S, Yang H, Li J. Prognostic impact of galectin-3 in chronic kidney disease patients: a systematic review and metaanalysis. Int Urol Nephrol 2019;51:1005-1011.

38. Hogas S, Schiller A, Voroneanu L, et al. Predictive value for galectin 3 and cardiotrophin 1 in hemodialysis patients. Angiology 2016;67:854-859. 